\title{
Cardiac Catheterisation for ST-Elevation Myocardial Infarction During COVID-19 in Singapore: Protocols and Recommendations
}

\section{Dear Editor,}

Patients with COVID-19 are at risk of developing acute myocardial infarction during the course of infection. A critical question is how are cardiac catheterisation laboratories managing ST-elevation myocardial infarction in patients with suspected/ confirmed COVID-19? In this brief clinical report, we described our early experience and highlighted a few key approaches.

\section{Introduction}

COVID-19 has been declared a pandemic by the World Health Organization (WHO) on 11 March 2020. Healthcare providers around the world are all facing an unprecedented situation for the first time, battling with ever-changing dynamics and enormous patient load. Older age at presentation and existing co-morbidities which include cardiovascular disease, diabetes mellitus, chronic respiratory disease, etc, are important predictors of mortality. ${ }^{1}$

COVID-19 patients are at risk of developing acute myocardial infarction (AMI) during the course of infection. A critical question is how are cardiac catheterisation laboratories (cath lab) managing ST-elevation myocardial infarction (STEMI) in patients with suspected/confirmed COVID-19? No robust consensus has been put forth by the interventional community so far. ${ }^{2,3}$ China physicians who were facing the first wave of COVID-19 patients had advocated thrombolysis as first-line therapy instead of primary percutaneous coronary intervention (PPCI). ${ }^{4}$

To date, Singapore has recorded a total of 38,296 confirmed cases of COVID-19 with 25 fatalities. Our institution is co-located with the National Centre for Infectious Disease (NCID) where most of the COVID-19 confirmed cases are hospitalised. PPCI is the first-line re-perfusion therapy for STEMI in Singapore due to the infrastructure in place. ${ }^{5}$ In this brief clinical report, we described our early experience in dealing with suspected/confirmed COVID-19 patients with STEMI in the cath lab.

\section{COVID-19 Cath Lab Preparation and Protocol}

A COVID-19 suspected case is defined as one who presents with an acute respiratory illness of any degree of severity with or without prior travel history to the high-risk countries. There are marked overlaps in terms of clinical presentations as well as radiologic findings for both COVID-19 and acute coronary syndrome. ${ }^{6}$

COVID-19 patients with lung involvement may require oxygen therapy. Those with concomitant AMI may suffer pulmonary congestion and develop acute respiratory distress.

Since COVID-19 is transmitted primarily through aerosol, healthcare providers in the cath lab are at higher risk of contracting the disease due to close patient contact. Avoidance of aerosol generating measures such as nebulisation, non-invasive ventilation and high flow nasal oxygen therapy are important for staff protection. ${ }^{7}$

In our institution, we adopt a prophylactic early intubation strategy in the emergency room/ward for COVID-19 patient with STEMI if the patient demonstrated significant signs and symptoms suggestive of acute respiratory distress before transfer to the cath lab. We also advocate this strategy for those who have a high likelihood of respiratory deterioration (Killip Class II or above, supplemental oxygen therapy $>4$ litres/minute and chest $\mathrm{x}$-ray showing bilateral haziness).

By securing the airway through elective intubation, we feel this is much safer than emergency intubation if patient decompensates half-way during the PPCI procedure. ${ }^{8-11}$ In addition, STEMI patients are also prone to sudden cardiac arrest so securing the airway early in patients with high likelihood of clinical deterioration seems rational. The PPCI procedure could also be completed in a smooth manner while minimising risk of exposure to cath lab staff.

Our PPCI team consists of a cardiac interventionalist, 1 radiographer, 1 cardiac technician and 4 nurses. We devised a COVID-19 cath lab protocol to ensure the role of each personnel is clearly defined during the procedure. Figure 1 illustrates the workflow in our cath lab for suspected/confirmed COVID-19 cases. 
All personnel are N95 mask-fitted and had undergone preparatory dry runs. It is mandatory for the team to don a surgical cap, N95 mask, visor mask, disposable gown, sterile gloves and shoe covers before the procedure. There is a nurse designated runner who ensures the proper donning and doffing of PPE. There are separate areas for donning of PPE before the procedure and for doffing after completion of the procedure. Every cath lab will be strongly recommended to set up separate area/cubicle for donning and doffing of PPE. This will ensure proper waste disposal and avoid cross-contamination. All staff (except for the runner) is to remain in the angiographic suite and not allowed to enter the control room during the procedure to prevent cross-contamination between various areas of cath lab.

Security officers are activated via a dedicated route to accompany smooth transfer of patient. Housekeeping team is activated for terminal cleaning after the procedure. The cath lab is essentially a positive-pressure room. Portable fan devices with high-efficiency particulate air (HEPA) filtration are utilised to increase the effective air changes per hour to the angiographic room.

\section{Patient 1}

On 23 January 2020, a 62-year-old man presented to our emergency department with a 2-day history of shortness of breath. He was a visitor from Hebei province, China and had arrived in Singapore on 22 January 2020 . Hebei is approximately 900 kilometres from Wuhan, the epicenter of COVID-19 outbreak.

$\mathrm{He}$ was febrile with a body temperature of $38.2^{\circ} \mathrm{C}$, blood pressure of $119 / 59 \mathrm{mmHg}$, respiratory rate of 22 breaths per minute and oxygen saturation of $90 \%$ on room air. Lung examination revealed bilateral basal crepitations. Chest $\mathrm{x}$-ray showed bilateral pulmonary congestion and air space opacities (Fig. 2). He also had a history of diabetes mellitus and hypertension. Initial blood investigations revealed neutrophilia (7.1 $\left.\times 10^{\wedge} 9 / \mathrm{L}\right)$, lymphopenia $\left(0.95 \times 10^{\wedge} 9 / \mathrm{L}\right)$, and elevated C-reactive protein $(115.1 \mathrm{mg} / \mathrm{L})$. Troponin was

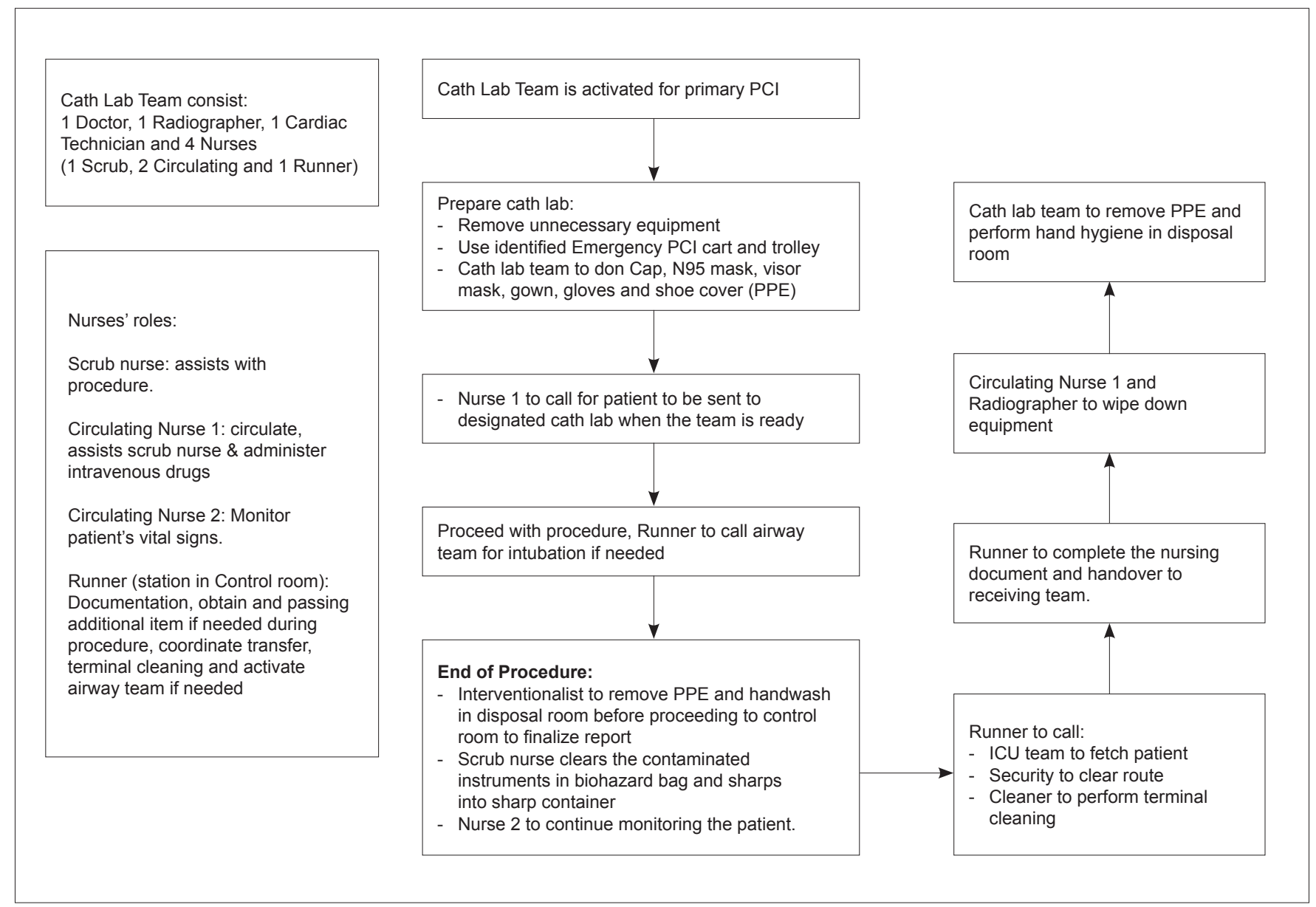

Figure 1. Workflow for management of patients with suspected/confirmed COVID-19 patients 


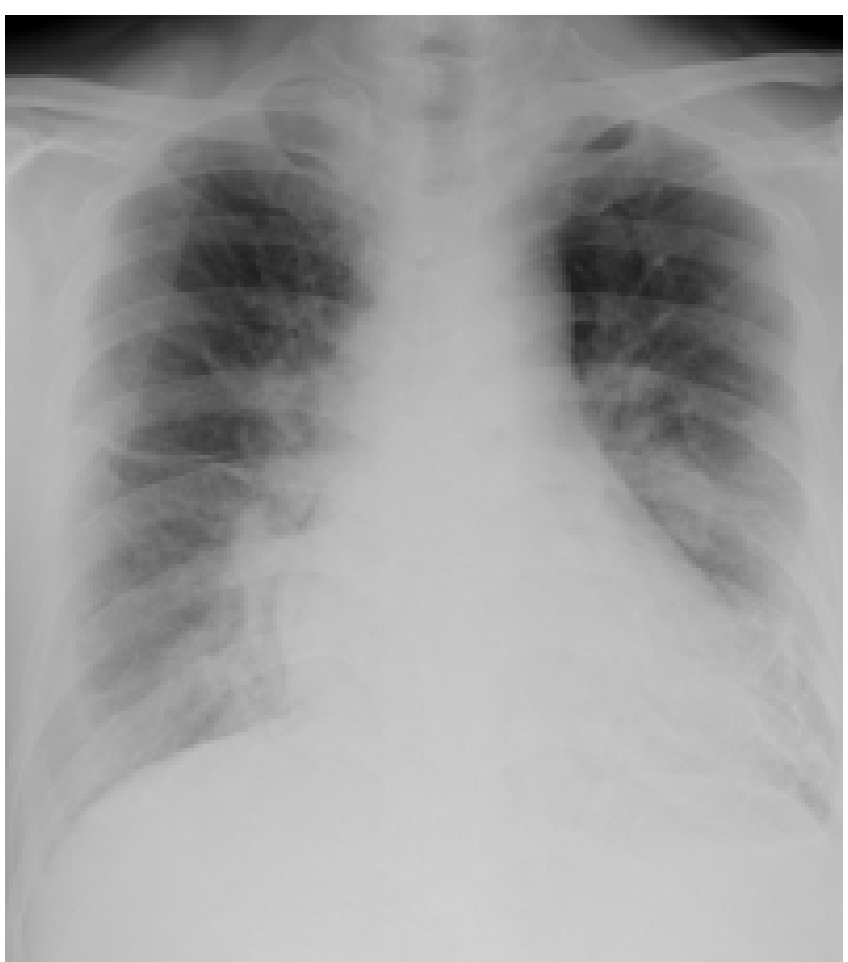

Figure 2. Chest radiograph (Postero-anterior view), January 2020

elevated at $3808 \mathrm{ng} / \mathrm{L}$ (normal range $0-18$ ) and electrocardiogram (ECG) revealed sinus bradycardia with $\mathrm{T}$ inversion in lead III. He was treated as nonSTEMI with concomitant heart failure and was given dual anti-platelet therapy, low-molecular weight heparin and diuretics.

Given the patient's travel history, the NCID was immediately notified. The patient was labeled as a "suspected COVID-19 case". Specimens were collected in accordance to international guidelines.

On day 2 of admission, he became more dyspnoeic and ECG showed ST elevation in the inferior leads with transient complete heart block. Before he was brought to our cath lab for PPCI, the patient was electively intubated by the intensivist with the aid of powered air-purifying respirator (PAPR). Our COVID-19 cath lab protocol was activated and the cath lab staff were in full PPE gear before the arrival of the patient. Coronary angiogram showed triple vessel disease with acute occlusion of right coronary artery. Successful PPCI was performed with two drug eluting stents implanted in the occluded vessel.
He was subsequently transferred to the intensive care unit (ICU) of NCID and was successfully extubated on day 4 of ICU stay. Subsequent tests for SARS-CoV2 PCR specimens were negative. The patient recovered well and was discharged on day 9 of hospitalisation.

\section{Patient 2}

On March 1, 2020, a 73-year-old man presented to our emergency department with a 4-day history of shortness of breath. His spouse was earlier diagnosed with COVID-19. He had a known history of hypertension and chronic kidney disease. Physical examination revealed a body temperature of $36.9^{\circ} \mathrm{C}$, blood pressure of $132 / 63 \mathrm{mmHg}$, heart rate of 60 beats per minute, respiratory rate of 16 breaths per minute and oxygen saturation of $98 \%$ on ambient air. Lung auscultation was normal and chest x-ray (Fig. 3) showed no gross abnormalities.

Computed tomographic (CT) scan of thorax on 2 March 22020 showed subpleural and peri-bronchial irregular foci of ground glass consolidation in both lungs (Fig. 4). He developed intermittent fevers but blood cultures were negative. Collected specimens from multiple sites for SARS-CoV2 PCR were consistently negative.

He became more dyspnoeic on day 9 of hospitalisation. Chest $\mathrm{x}$-ray showed worsening air space opacities (Fig. 3). In view of his escalating oxygen requirement, he was electively intubated. Endotracheal tube aspirate was positive for SARS-CoV2 PCR on the same day.

Kaletra (lopinavir/ritonavir) and subcutaneous interferon beta were initiated for him.

On 11 March 11 2020, he complained of mild chest discomfort. ECG showed $1.5 \mathrm{~mm}$ ST elevation in the inferior leads. Our COVID-19 cath lab protocol was activated before transfer to the cath lab. Urgent coronary angiogram showed triple vessel disease (95\% stenosis in mid-left anterior descending artery, diffuse disease in left circumflex artery and 70\% stenosis in right coronary artery) with the thrombolysis in myocardial infarction (TIMI) 3 flow distally. The plan of management was to refer him for consideration of coronary artery bypass grafting once his acute issues have resolved.

Troponin was elevated at $6727 \mathrm{ng} / \mathrm{L}$ (normal range $0-18$ ). He was deemed to have type $2 \mathrm{MI}$ and was treated with dual anti-platelet therapy and low 

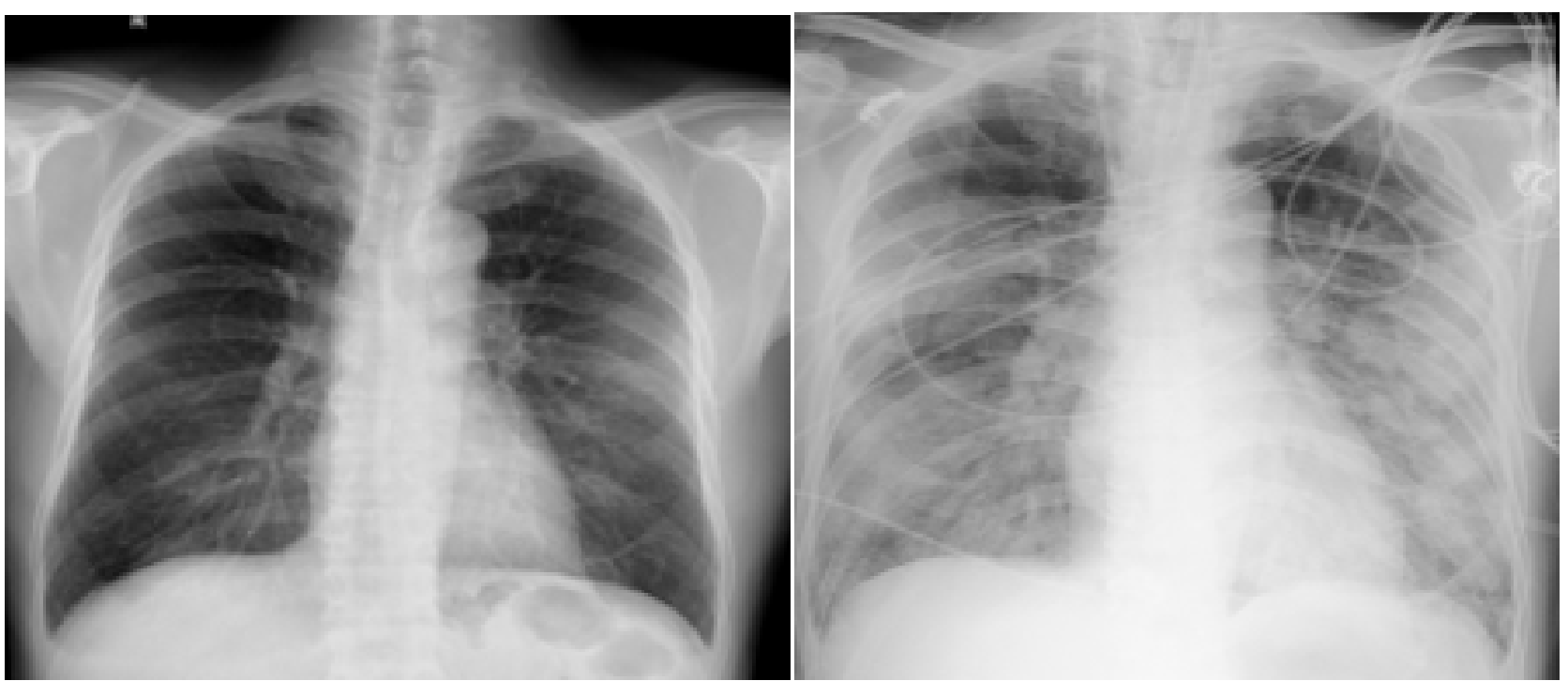

Figure 3. Chest radiograph (Left, day 1 of hospitalisation; Right, after intubation on day 9)

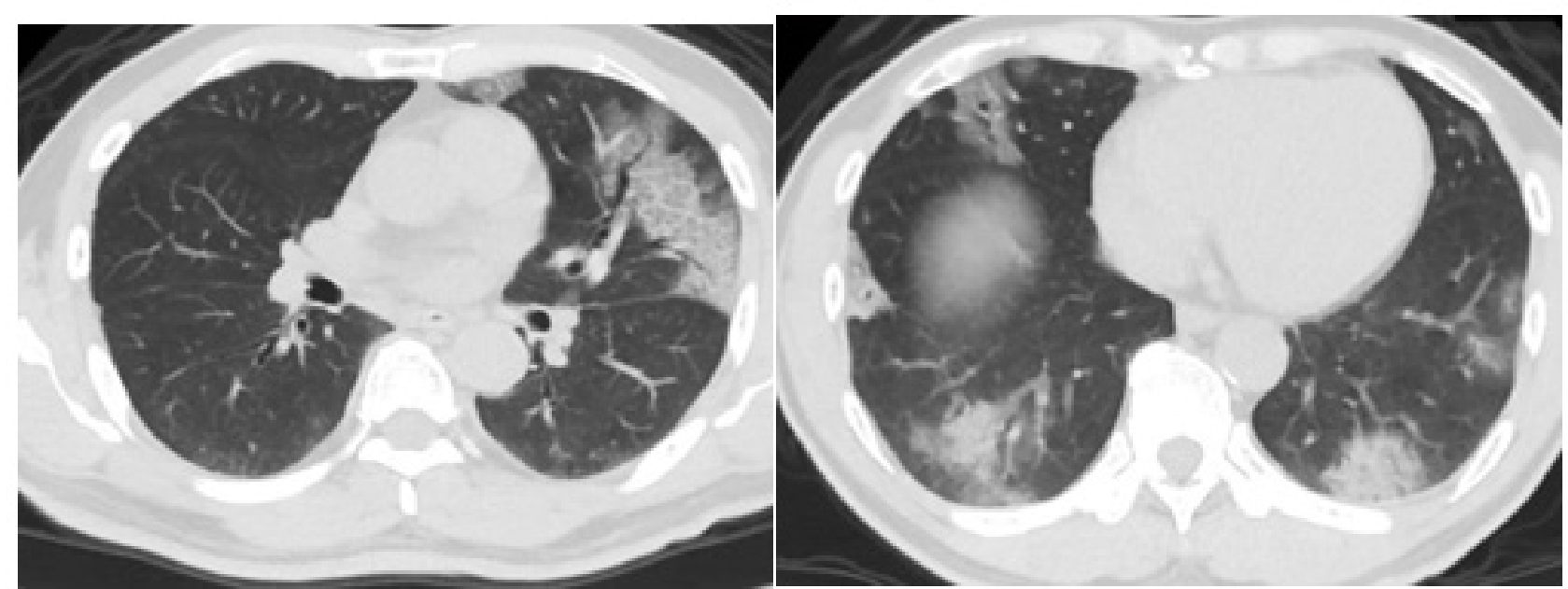

Figure 4. Computed tomographic axial scan of thorax, March 2020

molecular weight heparin. He underwent tracheostomy in view of prolonged ventilation and required intensive rehabilitation as of 20 April 2020.

\section{Discussion}

Currently, there is no robust consensus on how to treat COVID-19 patients with STEMI., . $^{3,4}$ Many physicians advocate thrombolysis as the "safer option" based on the Sichuan experience. ${ }^{5}$ Thrombolysis may not be suitable for some of the COVID-19 patients as they may have conditions like acute myocarditis, stress-induced cardiomyopathy, etc, which can mimic a true STEMI. There is also an inherent bleeding risk (especially intracranial) with thrombolysis, hence it may not be a safer option.

Due to the following concerns, PPCI remains the first-line reperfusion therapy in our institution. There are 
few hours. Priority must be given to STEMI patients and those with non-STEMI who are haemodynamically unstable as PCI procedures will be life-saving.

We share our preliminary experience and highlight a few key approaches:

(1) Adopting a standardised COVID-19 cath lab protocol for smooth workflow and staff safety. All cath lab staff must use maximal PPE during the procedure and re-establish a safe environment when the case is over.

(2) We strongly advocate a prophylactic early intubation strategy if the COVID-19 and STEMI patient demonstrated significant signs and symptoms of acute respiratory distress or has a high likelihood of respiratory deterioration. Clinical judgement is paramount in every case as we do not recommend routine intubation.

(3) An airway team with full PPE and powered air-purifying respirator (PAPR) should ideally be on standby for a stable COVID-19 and STEMI patient (who was not intubated prophylactically) when they undergo PPCI.

The COVID-19 pandemic has greatly impacted the health care system worldwide and our sharing of best practice may not be applicable in every institution. Healthcare providers have to grapple with inherent risk of infection while delivering the standard of care with some hospitals running short of manpower, PPE, ventilators and intensive care units. In our institution, we were fortunate not to have resource limitation of equipment and manpower. Ideally, future cath lab design should include a dedicated angiographic suite with negative pressure capability, designated donning and doffing areas. In a resource limited setting, minor modifications such as improving air exchanges, installing dedicated HEPA filters and proper disinfection processes are all essential.

Nevertheless, WHO has called for everyone to innovate and learn during this COVID-19 pandemic. All of us have to answer the call, meet the challenges in the cath lab with good teamwork and adopt the principle of maximum protection.

\section{REFERENCES}

1. Zhou F, Yu T, Du R, Fan G, Liu Y, Liu Z, et al. Clinical course and risk factors of mortality of adult inpatients with COVID-19 in Wuhan, China: a retrospective cohort study. Lancet 2020; $395: 1054-62$.

2. Welt FGP, Shah PB, Aronow HD, Bortnick AE, Henry TD, Sherwood MW, et al. American College of Cardiology's (ACC) Interventional Council and the Society of Cardiovascular Angiography and Intervention (SCAI). Catheterization laboratory considerations during the coronavirus (COVID-19) pandemic: from ACC's Interventional Council and SCAI. J Am Coll Cardiol 2020; 75: 2372-5.

3. Szerlip M, Anwaruddin S, Aronow HD, Cohen MG, Daniels MJ, Dehghani P, et al. Considerations for cardiac catheterization laboratory procedures during the COVID-19 — pandemic perspectives from the society for cardiovascular angiography and interventions emerging leader mentorship (SCAI ELM) members and graduates. Catheter Cardiovasc Interv 2020; 10: 1-12.-

4. Zeng J, Huang J, Pan L. How to balance acute myocardial infarction and COVID-19: the protocols from Sichuan Provincial People's Hospital. Intensive Care Med 2020;46:1111-3.

5. Dmitriy K, Tan HC, Poh KK. Management of acute st-elevation myocardial infarction: reperfusion options. Ann Acad Med Singapore 2010;39:927-33.

6. Shi H, Han X, Jiang N, Cao Y, Alwalid O, Gu J, et al. Radiological findings from 81 patients with COVID-19 pneumonia in Wuhan, China: a descriptive study. Lancet 20:425-34.

7. Wax RS, Christian MD. Practical recommendations for critical care and anesthesiology teams caring for novel coronavirus (2019-nCoV) patients. Can J Anesth 2020;67:568-76.

8. Goh KJ, Choong MCM, Cheong EH, Kalimuddin S, Wen SW, Phua GC. Rapid progression to acute respiratory distress syndrome: review of current understanding of critical illness from covid-19 infection. Ann Acad Med Singapore 2020;49:108-18.

9. Zuo MZ, Huang YG, Ma WH, Xue ZG, Zhang JQ, Gong YH, et al. Expert recommendations for tracheal intubation in critically ill patients with novel coronavirus disease 2019. Chin Med Sci J. 2020;35:105-9.

10. Peng PWH, Ho PL, Hota SS. Outbreak of a new coronavirus: what anaesthetists should know. Br J Anaesth. 2020;124:497-501.

Shiun Woei Wong, ${ }^{1} M R C P$,

Jin Hyun Lee, ${ }^{1} M R C P$, Li Min Ling. ${ }^{2}{ }_{M R C P}$,

Hwei Hian $\underline{\text { Tan }},{ }^{1}{ }_{B S C N}$, Hee Hwa $\underline{\text { Ho, }}$, FFACC

${ }^{1}$ Department of Cardiology, Tan Tock Seng Hospital, Singapore

${ }^{2}$ National Centre for Infectious Diseases, Singapore

Address for Correspondence: Dr Shiun Woei Wong, Department of Cardiology, Tan Tock Seng Hospital, 11, Jalan Tan Tock Seng, Singapore 308433 Email: shiunwoei@gmail.com 